\title{
Image Registration using PSO and APSO: A Comparative Analysis
}

\author{
Jitendra Pramanik \\ M.Tech Scholar \\ Dept. of ECE, CUTM \\ Bhubaneswar, Odisha, INDIA
}

\author{
Sunita Dalai \\ Asst. Professor \\ Dept. of ECE, CUTM \\ Bhubaneswar, Odisha, INDIA
}

\author{
Debaraj Rana \\ Asst. Professor \\ Dept. of ECE, CUTM \\ Bhubaneswar, Odisha, India
}

\begin{abstract}
Image registration, also known as matching or warping can be defined as the process of aligning two or more images. Image registration is a crucial step for image analysis in which valuable information is conveyed in more than one image, ie.images acquired at different times, from distinct viewpoints or by different sensors can be complementary .This research paper proposes a recently developed new variant of Particle Swarm Optimization (PSO) called Accelerated Particle Swarm Optimization (APSO) in image registration application.. APSO is simpler to implement and it has faster convergence when compared to the standard PSO (SPSO) algorithm. Here the extraction of interest point area from an image is optimized using Accelerated Particle swarm Optimization as well as PSO and a comparative analysis has been shown.
\end{abstract}

\section{Keywords}

Image Registration, Accelerated Particle swarm Optimization, Particle Swarm Optimization, DWT.

\section{INTRODUCTION}

Image registration is the fundamental image processing technique to determine geometrical transformation that gives the most accurate match between reference and floating images. Image registration can also be defined as a degree of similarity between images of same objects or scene which are taken at different times, different view angles or from different sensors [1]. Image registration has a variety of application in the field of military automatic target recognition remote cartography, computer vision, image fusion, medical imaging, and astrophotography.

The image registration process generally consists of the following steps

1. Feature extraction: It identifies the effective features of both source and target image e.g. edges, intersection of lines, and regions.

2. Feature matching: Establishment of a relationship between the features extracted from the two images

3. Mapping function building: Determination of mapping function parameter for registration using extracted features.

4. Image registration: Geometrical transformation and resampling the source image according to the mapping function established in step (3).

There are many numbers of image registration algorithms present which can be divided into two categories.

\section{$>$ Feature-based methods}

Area based methods are classical image registration methods. In area based method optimization algorithm are being used for searching the transformation model between two images by taking either a predefined size of window or the whole image. For example Cross correlation matching and least square matching are the most commonly used area based methods [1-2].

The feature-based methods utilize the salient features instead of image intensities to determine the transformation model between the two input images. These methods have higher computation efficiency than the area-based method. So the feature-based methods are extensively used to align images having complex geometric distortions [1, 3]. Interest point matching in images has been a difficult aspect in image registration and remains the subject of much research within the communities of remote sensing, computer vision systems, pattern recognition, and medical image processing. [1].

In remote sensing, area-based algorithms are normally suitable for open terrain areas, but the feature-based approaches can provide more accurate results in urban areas. No single technique performs well in both circumstances [4]. Both algorithms have their own unique strengths and weaknesses. Neither type of algorithm can avoid the problem of dealing with ambiguity in smooth (low texture) areas. Feature-based algorithms face the additional problem of the effect of outliers (points with no correspondences) on the results [5].

The accuracy image registration algorithm is generally affected by the segmentation and feature extraction algorithm. Therefore researching and finding a more accurate and faster image registration algorithm is a very important. The advantage of a feature based method, where a matching algorithm is required between corresponding objects within the images, is with the pixel intensity. However this method is very sensitive to the error evolving in feature extraction and matching process [6].

In this paper image registration is implemented using PSO as well as APSO. For image registration features have been extracted by the help of wavelet transform. All the methods used as describe in the following sections. This paper organise as follows: Section 2 described about DWT and feature extraction, section 3 describe about optimization technique. Proposed method are presented in section 4 and the result and discussion is given in section 5 followed by conclusion in section 6 .

$>$ Area based method 


\section{DISCRETE WAVELET TRANSFORM}

The wavelet transform (WT) has a large number of applications in signal processing and image compression. Due to its multi-resolution nature, wavelet-coding schemes are especially suitable for applications where scalability and tolerable degradation are important [7]. The wavelet transform performed with the advantage of time frequency localization. Multi-resolution analysis, analyzes the signal at different frequencies with different resolutions.

Discrete wavelet transform is any wavelet transform for which the wavelets are discretely sampled with capability of capturing both frequency and location information. It provides spatial and frequency representations of the image and also applicable for feature extraction. It decomposes the input data into several layers of division in space and frequency and allows us to isolate the frequency components introduced by intrinsic deformations due to expression or extrinsic factors (like illumination) into certain sub-bands. There exists a variety of wavelet families depending on the choice of the mother wavelet [8]. In this paper we are using the DWT which is based on the features extracted from a Haar Wavelet Transform. The Haar wavelet transform is a widely used technique in case of DWT. It is a powerful technique for the multi-resolution decomposition of time series [9].

The concept behind discrete wavelet transform is that images processed are transformed to a multi difsferentiated format in which the image is decomposed into sub image of different spatial domain and independent frequency district. After DWT transform of original image, then it is decomposed into four different frequencies in which one is low-frequency components and the other three are high frequency components. Those frequency components are termed as LL, LH HL HH [8].

\begin{tabular}{|l|l|l|}
\hline $\mathrm{LL}_{2}$ & $\mathrm{LH}_{2}$ & \multirow{2}{*}{$\mathrm{LH}_{1}$} \\
\hline $\mathrm{HL}_{2}$ & $\mathrm{HH}_{2}$ & \\
\hline $\mathrm{HL}_{1}$ & $\mathrm{HH}_{1}$ \\
& & \\
\hline
\end{tabular}

Fig 1. A 2-level wavelet decomposition of an M x M-pixel image

As shown in figure 1 at each level of the wavelet decomposition, four new images are formed from the original image of MxM pixel. The size of these new images is reduced to $1 / 4$ of the original size, so the new size is $M / 2 \times M / 2$. The new images are named according to the filter that is applied in horizontal and vertical directions. For an example the LH image is a result of application of the low-pass filter in horizontal direction and high pass filter in the vertical direction. Hence the four images produced from each decomposition level are labeled as LL, LH, HL, and HH. The LL image is considered to be a reduced version of the original but retains most details. The DWT coefficients in LL components are called as Detail coefficients. The LH image contains information of horizontal edge features, while the HL contains information of vertical edge features. The $\mathrm{HH}$ have only the high frequency information . HH is very noisy .Therefore it is not useful for the registration process. The coefficients in LH, HL, HH are termed as the approximate coefficient. Hence in wavelet decomposition, the LL image is used for producing the next level of decomposition which is the stable component.[9-11].

\section{ACCELERATD PARTICLE SWARM OPTIMIZATION}

\subsection{Review of particle swarm optimization}

The standard particle swarm optimization (PSO) was developed by Kennedy and Eberhart in 1995 which is inspired from the swarm behaviour such as fish and bird schooling in nature [12-13]. Then PSO has generated much wider interests and became an exciting, expanding research subject named as swarm intelligence. PSO has been applied to variety of optimization, designing applications. There are more numbers of PSO variants are available. Many hybrid algorithms have evolved by combining PSO with existing algorithms. PSO searches the space of an objective function by adjusting the trajectories of individual agents, called particles, as the piecewise paths formed by positional vectors in a quasistochastic manner.[14]

The movement of a swarming particle consists of two major components: a stochastic component and a deterministic component. Each particle is attracted toward the position of the current global best $\mathrm{g}^{*}$ and its own best location $\mathrm{xi}^{*}$ in history, while at the same time it has a tendency to move randomly

$$
\begin{aligned}
& v_{i}^{t+1}=W^{t} \cdot v_{i}^{t}+c_{1} \cdot r_{1} \cdot\left(\text { pbest }_{i}^{t}-X_{i}^{t}\right)+c_{2} \cdot r_{2} \cdot\left(\text { gbest }^{t}-X_{i}^{t}\right) \\
& X_{i}^{t+1}=X_{i}^{t}+v_{i}^{t+1}
\end{aligned}
$$

wher ${ }^{\mathrm{e}} \mathrm{X}_{\mathrm{i}}^{\mathrm{t}}$ and $\mathrm{v}_{\mathrm{i}}^{\mathrm{t}}$ denote the position and velocity of $i^{\text {th }}$ particle at time instance $t, \mathrm{~W}^{\mathrm{t}}$ is inertia weight at $\mathrm{t}^{\text {th }}$ instance of time, $\mathrm{c} 1$ and $\mathrm{c} 2$ are positive acceleration constants, and $\mathrm{r} 1$ and $\mathrm{r} 2$ are random values generated in the range [0,1], sampled from uniform distribution. pbest $\mathrm{i}_{\mathrm{i}}$ the best solution of the $\mathrm{i}^{\text {th }}$ individual particle over its fight path, gbest is the best particle obtained over all generation[15,16]. The algorithm as follows:

\section{Algorithm for PSO based image registration \\ Create $\mathrm{P}$ number of particles.}

for Each Particle $\mathrm{i}=1$ to $\mathrm{P}$ to do

Initialize parameters and corresponding random velocities.

end for

while (Termination condition $\neq$ true) do

for each Particle $\mathrm{i}=1$ to $\mathrm{P}$ do

Extract Subwindows

Calculate objective functional value

//Set as the personal best solution of $i^{\text {th }}$

// particle achieved so far.

if $F\left(\left(I_{e}\right)_{i}\right)>F\left(\right.$ pbest $\left._{i}\right)$ then

pbest $_{i}=\mathrm{P}_{\mathrm{i}}$

$/ / \mathrm{P}_{\mathrm{i}}$ is the $\mathrm{i}^{\text {th }}$ particle

end if

//Set $\mathrm{g}_{\text {best }}$ as the global best solution achieved

// so far among all generation.

if $F\left(\left(I_{e}\right)_{i}\right)>F\left(\right.$ gbest $\left._{i}\right)$ then

gbest $=\mathrm{P}_{\mathrm{i}}$

end if

end for

for Each Particle $\mathrm{i}=1$ to $\mathrm{P}$ do

Update the velocity

Update the position 
end for

end while

\subsection{Accelerated particle swarm optimization}

The standard particle swarm optimization uses both the current global best $\mathrm{g}^{*}$ and the individual best $\mathrm{x}_{\mathrm{i}}$. The reason of using the individual best is primarily to increase the diversity in the optimum solutions; however, this diversity can be simulated using some randomness. Subsequently, there is no strong reason for using the individual best, unless the optimization problem of interest is highly nonlinear. Another version which could accelerate the convergence of the algorithm is to use the global best only. Thus, in the accelerated particle swarm optimization (APSO) [17-18], the velocity vector is generated by the formula

$$
\mathrm{v}_{\mathrm{i}}^{\mathrm{t}+1}=\mathrm{v}_{\mathrm{i}}^{\mathrm{t}+1}+\alpha^{*} \varepsilon^{+} \beta\left(\mathrm{g}^{*}-\mathrm{x}_{\mathrm{i}}^{\mathrm{t}+1}\right)
$$

where $\varepsilon$ is drawn from $\mathrm{N}(0,1)$ to replace the second term. The update of the position is simply

$$
\mathrm{x}_{\mathrm{i}}^{\mathrm{t}+1}=\mathrm{x}_{\mathrm{i}}^{\mathrm{t}+1}+\mathrm{v}_{\mathrm{i}}^{\mathrm{t}+1}
$$

Typically, $\alpha=0.1 \mathrm{~L}$ to $0.5 \mathrm{~L}$ where $\mathrm{L}$ is the scale of each variable, while $\beta=0.1$ to 0.7 is sufficient for most applications [17].

\section{PROPOSED METHOD}

The proposed algorithm aims of providing image registration using accelerated particle swarm optimization as well as standard PSO with a comparative study. The process of registration here is to find the target section in a source image. Here the process of registration is represented as an application to PSO and APSO, an optimization problem. As per the concept of PSO and APSO some random population has been selected which represents the coordinate of random pixel inside the image.

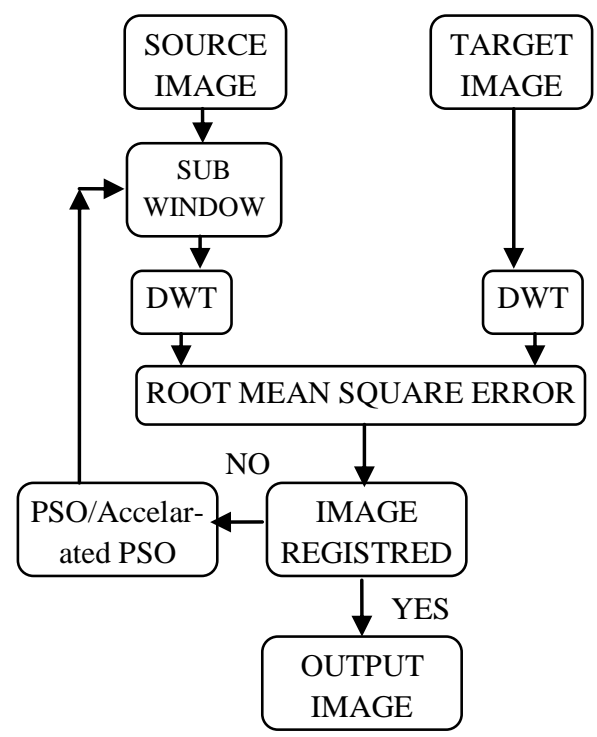

Fig 2. Block Diagram of image registration using PSO

The populations also include a size of window to be extracted. Then after formation of random population, each population will corresponds to a window inside the image. The windows which are the portion of the source image have some fitness value. The fitness value of all population is calculated using the DWT and RMS error. The RMS error has to found out between DWT of selected sub-windows and target block. In the initial generation of optimization technique we have to found out the best value. Then after updating the velocity and position new population will be generated. This process will go for a pre defined iterations number. Then the best value which is the 'gbest' will give the optimized windows.

After optimization using both APSO and PSO the performance graph has been plotted which represent no of iterations vs. fitness value. The position updation depends on velocities, which are selected within the range $[-3,3]$. The acceleration constant $\mathrm{c} 1$ and $\mathrm{c} 2$ values are taken as 2 in case PSO.

\section{RESULT ANALYSIS}

In the experiment the proposed method has been tested on varieties of image which are of different in sizes. The input images include scene image, satellite image, medical image images etc. In the experiment registered image is found through feature selection that has been implemented using wavelet transform and the optimization method. For the proposed method 'haar' wavelet has been choosen. The experimental result shows that APSO converge more rapidly as compared to PSO due to minimization of random movement of the particles.

Here some of the results we have shown which include the input image 'messi' (400 x500), 'vegetables' (400x400), 'satellite' $(400 \times 500) \quad, \quad$ 'tumor' $(350 \times 300)$ and 'itachi' $420 \mathrm{x} 600)$

The both methods PSO and APSO are conducted to the test images for registration, and that has been done by first finding the wavelet transform for feature extraction. The implementation of the proposed algorithm is done in MATLAB 7.8 using a computer with Intel i3 Processor $(2.20 \mathrm{GHz})$ and $4 \mathrm{~GB}$ RAM.

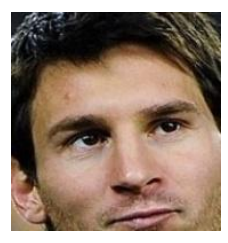

(a)

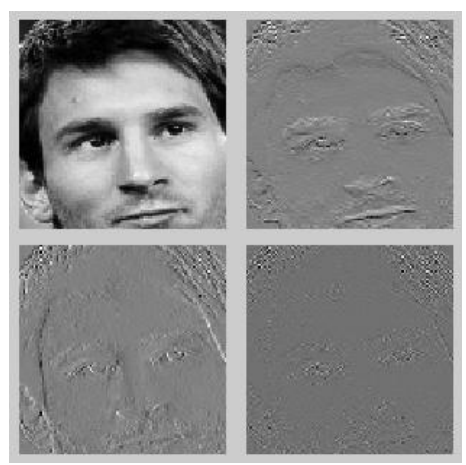

(c)
Fig 3. (a) Target Image (b) Gray Scale Image (c) 2D DWT

The following shows set of test images and some part of the images to be registered in figure 4 and 5 . 

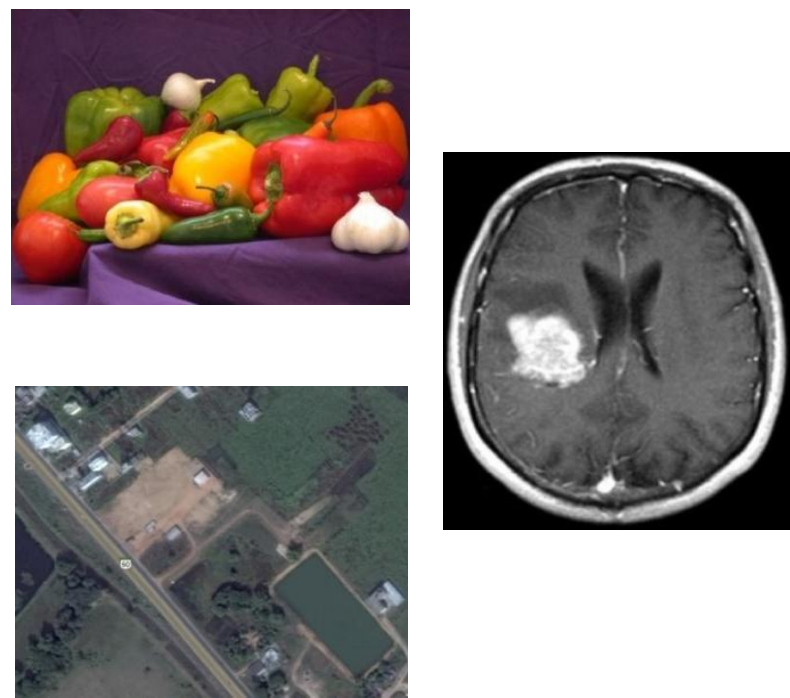

Fig 4. Set of Test Images
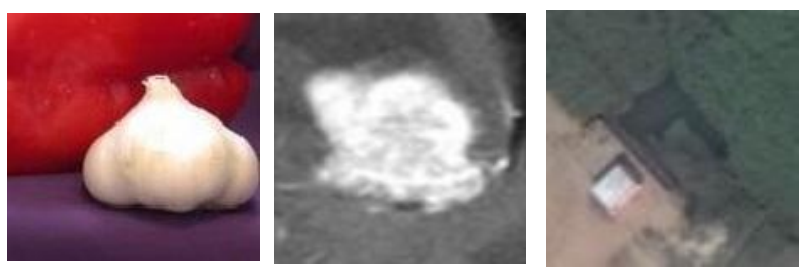

Fig.5 Set of Target Images

In this section, the experimental results for evaluating the efficiency of the proposed similarity measure are presented. Here the focus is on the use of proposed similarity measure technique for image matching and hence performing the image registration. In most of the cases both the methods perform better to give the registered image. But mostly APSO leads to the result quickly as compared to PSO.

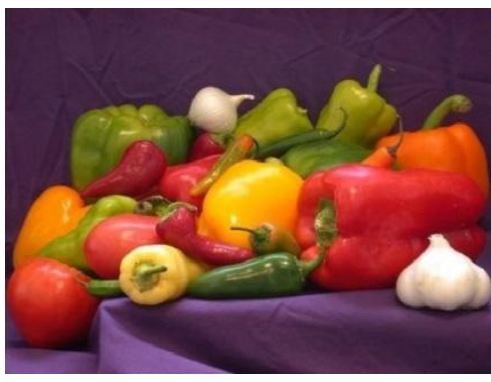

(a)

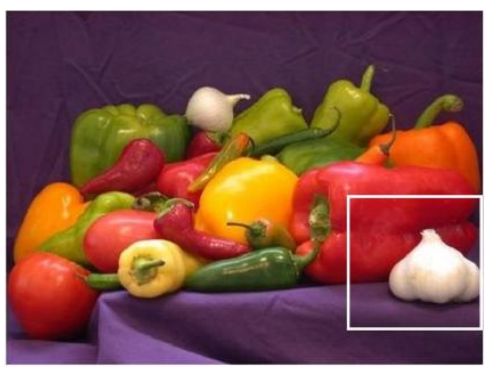

(c)

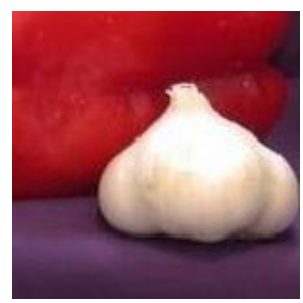

(b)

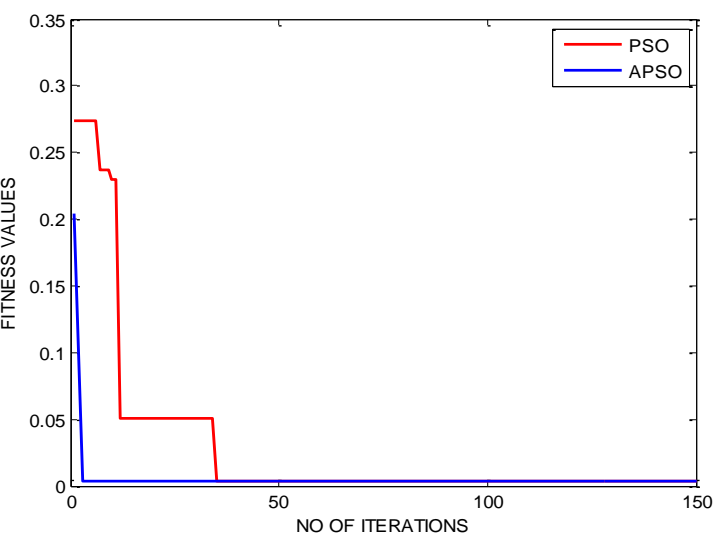

(d)

Fig.6 (a) Test Image (b) Target Image (c) Registered Image (d) Performance Plot

Here mostly 50 populations have been taken for both the PSO and APSO with 150 iterations to execute the result. Here some of resulted included in figure 6.

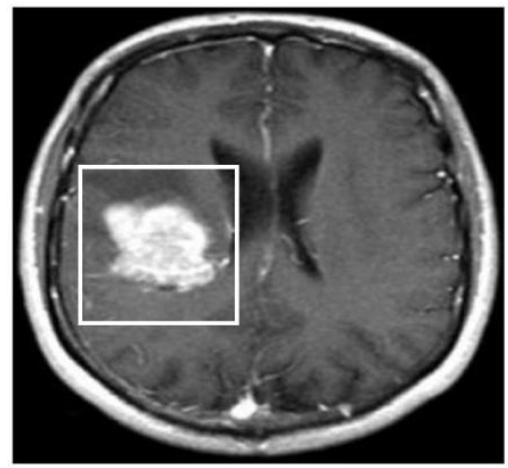

(a)

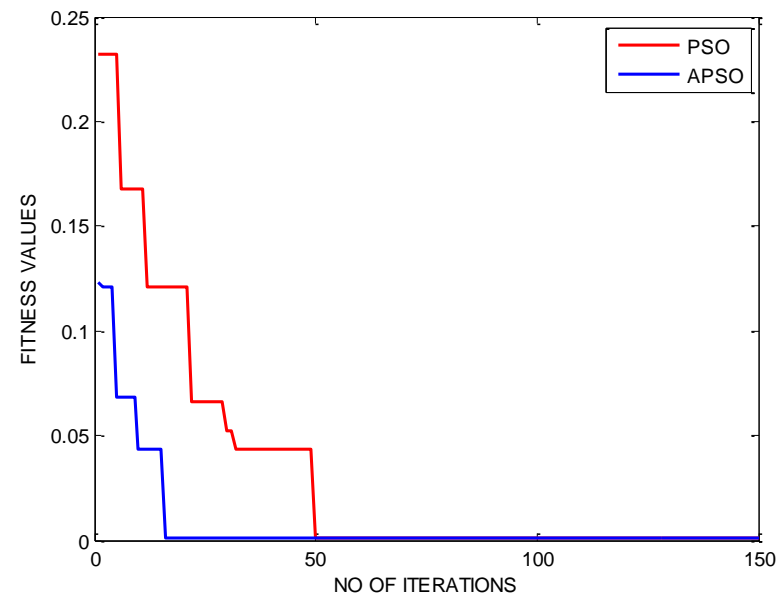

(b)

Fig.7 (a) Registered Image (b) Performance Plot

For the input image "tumor" in figure 7 and "satellite" in figure 8 , about 50 and 60 population has been taken for both PSO and APSO over 150 iteration respectively. In the test image "tumor" APSO converges in 20 iterations and PSO converges in 50 iterations, where as in case of 'satellite' both methods converges nearly same iteration, but APSO 
converges at 20 iterations and PSO converges at nearly 23 iterations.

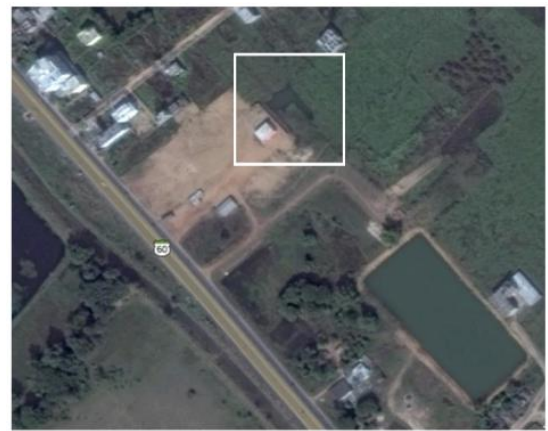

(a)

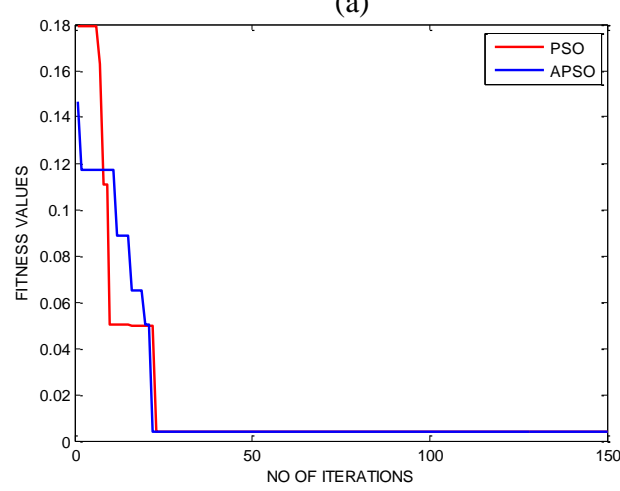

(b)

Fig.8 (a) Registered Image (b) Performance Plot

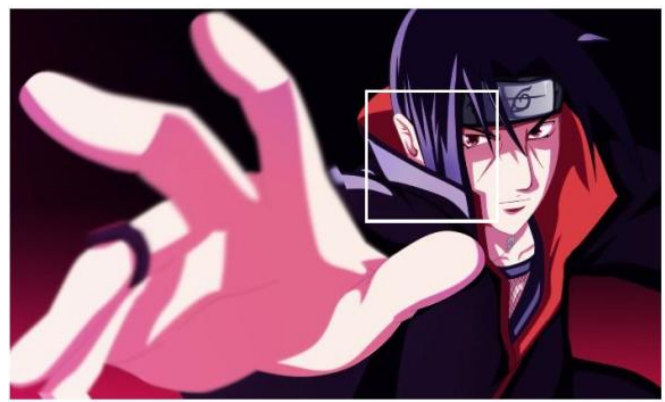

(a)

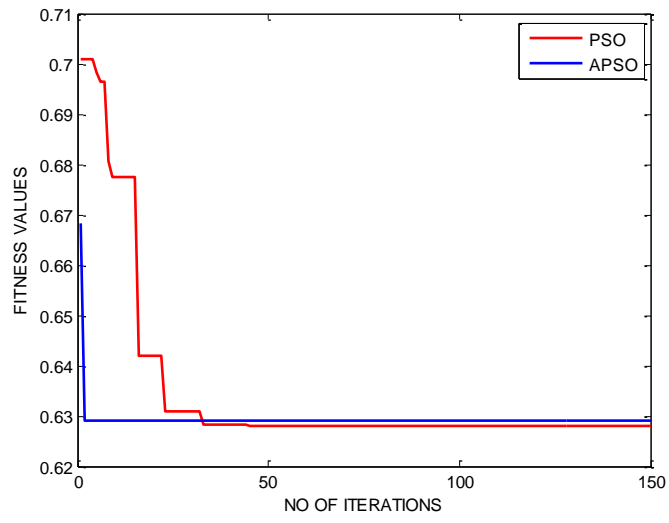

(b)

Fig.9 (a) Registered Image (b) Performance Plot
In the test image 'itachi' it has been shown in figure 9 (b) that APSO converges very early with in 10 generations where as PSO converges in 30 iterations.

The outputs have been tabulated for some test images with their number of iterations it takes for registration, with the corresponding dimension in the table 1 . The table shows the average iterations in PSO and APSO over a numbers of executions.

The table shows the image name with their dimension. The next two columns show the average iteration it takes for convergence to register in case of PSO and APSO respectively. The last column shows the number of initial population taken for execution.

Table 1. Number of iterations comparison

\begin{tabular}{|c|c|c|c|c|c|}
\hline $\begin{array}{c}\text { SI } \\
\text { No }\end{array}$ & Image & Dimension & PSO & APSO & POP \\
\hline 1 & tumor & $\mathbf{3 5 0} \times 300$ & 50 & 20 & 50 \\
\hline 2 & satellite & $400 \times 500$ & 23 & 20 & 60 \\
\hline 3 & vegetables & $400 \times 400$ & 35 & 10 & 50 \\
\hline 4 & messi & $400 \times 500$ & 50 & 28 & 70 \\
\hline 5 & itachi & $420 \times 600$ & 30 & 10 & 50 \\
\hline
\end{tabular}

\section{CONCLUSION}

In this paper image registration has been implemented using accelerated particle swarm optimization on various scene, satellite and medical images. The comparative analysis between the outputs of image registration obtained using APSO with the output of PSO is described. It is being observed from the performance graph that for given population taken for both the technique the APSO converges much faster compare PSO for same set of iteration. Experimental results on various images in registration shown by transparently overlapping source image with the extracted interest point area computed using PSO and APSO which provides an easy and efficient way of image registration.

The proposed work can be extended to optimize faster by implementing some others optimization techniques like Cuckoo Search and Water cycle algorithms (WCA) for image registration.

\section{REFERENCES}

[1] Ruhani B.Karani Dr.Tanuja K.Sarode “ Image Registration using Discrete Cosine Transform and Normalized Cross Correlation" International Conference \& Workshop on Recent Trends in Technology, (TCET) 2012.

[2] Barbara Zitova an d Jan Flusser "Image registration methods: a survey" Received 9 November 2001.

[3] Gang Hong, Yun Zhang "Combination of Feature-based and Area- based Image Registration Technique for High Resolution Remote Sensing Image" ieee international conference on Geoscience and Remote Sensing Symposium, 2007.

[4] M. Auer, P. Regitnig, and G. A. Holzapfel, "An automatic non rigid registration for stained histological sections," IEEE Trans. Image Process.,vol. 14, no. 4, pp. 475-486, Apr. 2005. 
[5] Hongjun Jia, Guorong Wu, Qian Wang, Minjeong Kim, and Dinggang Shen "Itree: fast and accurate image registration based on the combinative and incremental tree" international conference on Biomedical Imaging: From Nano to Macro, 2011.

[6] Nemir Al-Azzawi , Wan Ahmed K. Wan Abdullah “ MRI Monomodal Feature-Based Registration Based on the Efficiency of Multiresolution Representation and Mutual Information" American Journal of Biomedical Engineering 2012,2(3): 98-104.

[7] M. Alexander et al," Two-Dimensional Discrete Wavelet Transform on Large Images for Hybrid Computing Architectures: GPU and CELL", Euro-Par 2011 Workshops, Part I, LNCS 7155, pp. 481-490, 2012.

[8] Mei Jiansheng, Li Sukang1 and Tan Xiaomei "A Digital Watermarking Algorithm Based On DCT and DWT" Proceedings of the 2009 International Symposium on Web Information Systems and Applications (WISA'09) Nanchang, P. R. China, May 22-24, 2009, pp. 104-107

[9] Rabab M. Ramadan and Rehab F. Abdel - Kader "Face Recognition Using Particle Swarm Optimization-Based Selected Features" International Journal of Signal Processing, Image Processing and Pattern Recognition Vol. 2, No. 2, June 2009.

[10] Vidya V, Nazia Farheena, K Manikantan, S Ramachandran," Face recognition using threshold based DWT feature extraction and selective illumination enhancement technique", 2nd International Conference on Communication, Computing \& Security [ICCCS2012], Procedia Technology 6 ( 2012 ) 334 - 343

[11] D Rana, S Dalai, Bhawna, S Minz, N. Prasanna, T T Sahu," Comparative Analysis of Face Recognition using, DCT, DWT and PCA for Rotated faces", International
Journal of Scientific Research Engineering \& Technology (IJSRET), ISSN 2278 - 0882, Volume 3, Issue 5, August 2014, Page-852-857.

[12] J. Kennedy and R. C. Eberhart, Particle swarm optimization, in: Proc. of IEEE International Conference on Neural Networks, Piscataway, NJ. pp. 1942-1948 (1995).

[13] J. Kennedy, R. C. Eberhart, Swarm intelligence, Academic Press, 2001

[14] Web http://www.swarmintelligence.org/tutorials.php

[15] Apurba Gorai, Ashish Ghosh, "Gray-level Image Enhancement By Particle Swarm Optimization," 2009 World Congress on Nature \& Biologically Inspired Computing (NaBIC 2009)

[16] J. Pramanik, S. Dalai, D. Rana," Image Registration using Discrete Wavelet Transform and Particle Swarm Optimization", International Journal of Computer Science and Information Technologies (IJCSIT), Vol.6.Issue.2, March-2015, Page:1521-1525

[17] Yang, X. S., Deb, S., and Fong, S., (2011), Accelerated Particle Swarm Optimization and Support Vector Machine for Business Optimization and Applications, in: Networked Digital Technologies (NDT2011), Communications in Computer and Information Science, Vol. 136, Springer, pp. 53-66 (2011).

[18] K.Prajna, G.Sasi Bhushan Rao, K.V.V.S.Reddy, R.Uma Maheswari,“ A New Dual Channel Speech Enhancement Approach Based on Accelerated Particle Swarm Optimization (APSO)", I.J. Intelligent Systems and Applications, vol- 04, pp-1-10,2014 